\title{
DEVELOPMENT AND PRELIMINARY VALIDATION OF A DIAGNOSTIC SCORE FOR IDENTIFYING PATIENTS AFFECTED WITH ADULT-ONSET AUTOINFLAMMATORY DISORDERS
}

\author{
L. CANTARINI, O.M. LUCHERINI ${ }^{1}$, F. IACOPONI ${ }^{2}$, R. CIMAZ ${ }^{3}$, G. SIMONINI', \\ D. RIGANTE ${ }^{4}$, F. LAGHI PASINII ${ }^{5}$, C.T. BALDARI ${ }^{1}$, P.L. CAPECCHI ${ }^{5}$, M.G. BRIZI \\ and M. GALEAZZI
}

Interdepartmental Research Center of Systemic Autoimmune and Autoinflammatory Diseases, Unit of Rheumatology, Policlinico Le Scotte, University of Siena, Siena; 'Department of Evolutionary Biology, University of Siena, Siena; ' Department of Biomedical Science, University of Siena, Siena; ${ }^{3}$ Department of Paediatrics, Rheumatology Unit, Anna Meyer Children's Hospital and University

of Florence; ${ }^{4}$ Department of Pediatric Sciences, Università Cattolica Sacro Cuore, Rome; ${ }^{5}$ Interdepartmental Research Center of Systemic Autoimmune and Autoinflammatory Diseases, Unit of Immunological Sciences, Policlinico Le Scotte, University of Siena, Siena, Italy

\section{Received April 12, 2010 - Accepted September 7, 2010}

The first two authors contributed equally to this study

To date, the rate of detection of autoinflammatory gene mutations in patients suspected of having an autoinflammatory disorder is very low. However, most of these data refer to pediatric populations. The relative rarity and lack of information on adult-onset autoinflammatory diseases make it likely that mutations will be found in an even smaller percentage of cases. Our aim was to develop and validate a set of variables for predicting the risk that a given adult patient presenting with recurrent fever episodes carries mutations in the MEFV or TNFRSF1A genes, in order to increase the probability of obtaining positive results on genetic testing. One hundred and ten consecutive patients with a clinical history of periodic fever attacks were screened for mutations in the TNFRSFIA and the $M E F V$ genes. The mean age at disease onset was 27.85 years. Detailed information about each patient's family history, personal history, and clinical manifestations were retrospectively collected. A diagnostic score was constructed based on univariate and multivariate analysis in a randomly-selected dataset (training set; $n=40$ ). The score was validated on an independent set of the remaining patients (validation set; $n=70$ ). Age at onset (odds ratio [OR] 0.958, $P=0.050)$, positive family history of recurrent fever episodes (OR 5.738, $P=0.006)$, thoracic pain $(O R$ 7.390, $P=0.002)$, abdominal pain $(\mathrm{OR} 2.853, P=0.038)$ and skin involvement $(\mathrm{OR} 8.241, P=0.003)$ were independently correlated with a positive genetic test result. A diagnostic score was calculated using the linear combination of the estimated coefficients of the logistic model (cut off equal to 0.24 ) revealing high sensitivity (0.94), high specificity (0.94) and high accuracy $(0.94)$. We have identified variables that appear to be strongly related to the probability of detecting gene mutations in $M E F$ and $T N F R S F 1 A$ in adults, thus improving the evaluation of patients with suspected autoinflammatory disorders.

Key words: autoinflammatory diseases, diagnostic criteria, tumor necrosis factor receptor-associated periodic syndrome (TRAPS), familial Mediterranean fever (FMF) 
Familial Mediterranean fever (FMF) (Mendelian Inheritance in Man; MIM no.\#249100) and tumor necrosis factor receptor-associated periodic fever syndrome (TRAPS; MIM no. \#142680) are respectively the most common autosomal recessive and autosomal dominant monogenic autoinflammatory disorders, and are caused by mutations of genes encoding proteins that are involved in the regulation or activation of the inflammatory response (1).

Both FMF and TRAPS are characterized by recurrent episodes of fever variably associated with abdominal and chest pain, arthralgias and/or arthritis, cutaneous manifestations and lymphadenopathy. FMF attacks typically last from 1 to 3 days, while TRAPS attacks usually last more than 1 week. Overlapping symptoms complicate their differential diagnosis. Both conditions are characterized by disease flares separated by symptom-free intervals of variable duration with complete normalization of acute phase reactants.

The genes responsible for FMF and TRAPS are respectively the $M E F V$ gene, which encodes a protein called pyrin (2-3), and the TNFRSFIA gene, which encodes the 55-kD receptor for tumor necrosis factor (TNF)- $\alpha$ (4). Mutations causing FMF are present at a very high frequency in several populations, and the disease is most prevalent in the Mediterranean basin and Middle East (5), whereas TRAPS shows no ethnic predilection. Both diseases are more frequently diagnosed in the pediatric population, but FMF onset in adulthood, (6-7) and adult-onset TRAPS (8) have also been described.

To date, the rate of detection of autoinflammatory gene mutations in patients suspected of having an autoinflammatory disorder is very low, less than $20 \%$ in case series. However, most of these data refer to pediatric populations (9-10). The relative rarity and lack of information on adult-onset autoinflammatory diseases make it likely that mutations will be found in an even smaller percentage of cases. We are currently screening for possible autoinflammatory diseases in our Rheumatology Department, and are monitoring predominantly adult patients with genetically confirmed diagnoses. We have developed and provided preliminary validation for a set of variables that may predict the probability that a given adult patient presenting with recurrent fever episodes carries mutations in the $M E F V$ or TNFRSF $1 A$ genes, with the aim of increasing the probability of obtaining positive results with genetic testing.

\section{MATERIALS AND METHODS}

\section{Patients}

The study was conducted using data from 2 patient samples. A training sample was randomly selected from the initial screening group and used to create the diagnostic model. A separate validation sample was then used to validate the diagnostic model in an independent patient population.

The training and validation sets were randomly selected from among all patients meeting the inclusion criteria; each patient had the same probability of being included in the training or validation group.

All patients enrolled were seen between November 2007 and June 2009 at our Institution, and were part of a larger cohort of 487 patients treated for recurrent periodic fever attacks. Of these, 216 were excluded from the present study for one or more of the following reasons: 1) lack of complete clinical information 2) not meeting the inclusion criteria. In addition, the results of a genetic analysis of the two genes under study (MEFV, TNFRSF 1A), and complete clinical information were available for 110 of the remaining 271 patients. Detailed information about family history, personal history, and clinical manifestations were collected retrospectively.

Criteria for inclusion in the screening group were: 1) periodic fever attacks of unknown origin, with fever- and symptom-free intervals associated with normal levels of acute-phase reactants, and 2) one or more of the following symptoms present during fever attacks: abdominal pain, chest pain, Lymphadenopathy (documented by imagine techniques: echotomography and/or magnetic resonance imaging and/or high resolution computed tomography which were performed in order to role out underlying disorders), splenomegaly (echotomographic evaluation), cutaneous manifestations, musculoskeletal involvement, oral aphtosis, ocular involvement (periorbital oedema and/or conjunctivitis), orchitis. A total of 110 consecutive patients were enrolled in the screening group, 40 of whom were randomly entered in the training sample and 70 of whom were randomly entered in the validation sample.

For FMF, homozygous and compound heterozygous patients were considered to be genetically positive, while for TRAPS, heterozygous patients were considered to be genetically positive. All patients were Caucasians, of Italian ancestry. The study was approved by the Ethics Board of the Azienda Ospedaliera Universitaria Senese, 
Siena, Italy. Each patient had previously provided written consent for genetic testing, in accordance with the Helsinki Declaration and local Ethics Committee regulations, and also provided written consent for inclusion in the study.

\section{DNA extraction}

Mononuclear cells were purified from the peripheral blood of healthy donors and patients (with informed consent) by density gradient centrifugation on FicollPaque (Amersham Biosciences, Buckinghamshire, UK). Genomic DNA was isolated from the peripheral blood lymphocytes of patients and healthy controls using QIAamp DNA mini Kit (Qiagen, Hilden Germany).

\section{Genomic DNA amplification and mutation detection}

The TNFRSFIA gene exons 2, 3, 4 and 6, which encode the extracellular domain of the $55 \mathrm{kD}$ receptor for TNF- $\alpha$, and the $M E F V$ gene exons 2, 3, 5 and 10, where the majority of known mutations are found (11), were amplified by polymerase chain reaction (PCR) using Expand High Fidelity PCR System (Roche, Germany). PCR products were purified using the Wizard SV Gel and PCR Clean-Up System (Promega, Madison, WI, USA). Sequencing was carried out on the ABI 3730 DNA analyzer (Bio-Fab Research srl, Italy) using the same primers as those used for PCR.

\section{Statistical methods}

The primary aim of diagnostic score construction was to distinguish patients who were likely to have positive results on genetic testing from those who were likely to test negative, and therefore would not need to undergo genetic testing. As a secondary and exploratory objective, we attempted to assess the most logical order in which the genetic tests should be performed in patients who, after the first step, are determined to be at high risk.

The data are expressed as absolute frequencies and percentages (\%). The association between the variables under consideration and the presence of mutations (the outcome) was evaluated by $t$ test or chi square test, depending on the type of the variable (numerical or categorical). To optimize the model's predictive capacity, the original matrix was randomly divided, by a random number generator, into two sub-matrixes (learning and testing) and all the candidate variables were arranged in a dichotomous manner. Univariate logistic regression analysis was performed to identify the variables that were significantly associated with positive genetic test results. Variables yielding $p$ values of less than 0.05 were entered into a multivariate logistic analysis in which positivity of the genetic test was the dependent variable. Stepwise forward-backward logistic regression was used in the learning set for the inclusion of variables.
The minimization error was evaluated with receiver operating characteristic (ROC) curves, which assessed the performance of the model in the learning and training set. Model parameters were estimated in the learning set, and predictive performance evaluation was done in the testing set. Odds ratios (OR) and $95 \%$ confidence intervals ( $95 \%$ CI) were calculated for each risk factor as exponential of variable coefficients (beta). The risk classes were constructed through classification criteria based on sensitivity and specificity. In particular, the cut-off value suggested by ROC analysis was defined as the point that gave an optimal level of sensitivity and specificity.

In order to determine which genetic test should be performed first, the probability that a patient belongs to a particular risk class was calculated based on values for the independent variables. For this purpose, the diagnostic score was calculated by assigning a weight to each variable according to the beta coefficients estimated by the logistic model and then combining them in a linear fashion. Only symptoms that were correlated at a 5\% significance level with a specific disease category in the univariate analysis by chi square test were included as continuous variables in the regression tree analysis. A $\mathrm{p}$ value $<0.05$ (2-tailed) was considered statistically significant. All statistical analyses were performed with the software packages SPSS version 13.0 (SPSS, Inc., Chicago, Il) and MatLab version 7.0.1 (The MathWorks Inc. Natick, Ma).

\section{RESULTS}

At the time of genetic testing, the majority of the patients $(82.0 \%)$ were over the age of 16 (mean 32.26 years [range 3-68 years]). The mean age at disease onset was 27.85 years (range 2-65 years). A total of 92 patients did not have mutations in the regions of the 2 genes examined, whereas 18 patients were identified as having homozygous or compound heterozygous mutations in $M E F V$ (6 patients; 2 in the training set and 4 in the validation set) or heterozygous mutations in TNFRSFIA (12 patients; 5 in the training set and 7 in the validation set). Table I summarizes the frequencies of clinical manifestations in the patients with positive genetic tests. Since none of the patients enrolled $(0 / 110)$ showed orchitis, orchitis was not included in the statistical evaluation.

After the screening procedure was completed, 40 of the 110 patients were included in the training set. Table II shows the findings of the univariate logistic regression analysis (with positive genetic test results 
Table I. Clinical characteristics of patients with positive results on genetic testing.

\begin{tabular}{lccc}
\hline Independent variable & TNFRSFIA $(\mathrm{n}=12)$ & MEFV $(\mathrm{n}=6)$ & No mutations (n=92) \\
\hline Abdominal pain & $5(41.66 \%)$ & $4(66.66 \%)$ & $16(17.39 \%)$ \\
Thoracic pain & $11(91.66 \%)$ & $3(50 \%)$ & $28(30.43 \%)$ \\
Skin Rash & $4(33.33 \%)$ & $5(83.33 \%)$ & $14(15.22 \%)$ \\
Musculoskeletal involvement & $6(50 \%)$ & $5(83.33 \%)$ & $63(68.48 \%)$ \\
Ocular involvement & $1(8.33 \%)$ & 0 & $19(20.65 \%)$ \\
Periorbital edema & $1(8.33 \%)$ & 0 & $8(8.70 \%)$ \\
Headache & $1(8.33 \%)$ & $1(16.66 \%)$ & $19(20.65 \%)$ \\
Lymphadenopathy & $1(8.33 \%)$ & $3(50 \%)$ & $21(22.83 \%)$ \\
Age at onset $>16$ yrs) & $9(75 \%)$ & $2(33.33 \%)$ & $78(84.78 \%)$ \\
Positive family history & $6(50 \%)$ & 0 & $9(9.78 \%)$ \\
Duration of fever $(>7$ days) & $4(33.33 \%)$ & 0 & $46(50 \%)$ \\
Oral aphtosis & $1(8.33 \%)$ & $1(16.66 \%)$ & $20(21.74 \%)$ \\
Splenomegaly & $2(16.66 \%)$ & $2(33.33 \%)$ & $22(23.91 \%)$ \\
Orchitis & -- & -- & -- \\
\hline
\end{tabular}

as the dependent variable) and the variables retained in the final multivariate model. All symptoms were studied in univariate analysis as binary variables (presence or absence of symptoms). The ROC curve for the training set, with a cutoff equal to 0.24 , indicated a sensitivity of 0.912 , a specificity of 0.960 and an accuracy of 0.955 (Fig. 1A). This cutoff value guaranteed high sensitivity and good specificity. A total of 70 patients were enrolled in the validation set. The ROC curve in the validation set indicated a sensitivity of 0.944 , a specificity of 0.939 and an accuracy of 0.938 (Fig. 1B).

The variables retained in the final model were recoded according to the frequency (never, sometimes, often, always) of clinical manifestations (abdominal pain, chest pain, skin rash) and according to risk class (age at onset, positive family history) in order to achieve the best fit (Table III). The codes (never, sometimes, often, and always) refer to each episode of fever and not to the time between attacks. After re-coding, a diagnostic score was calculated using the linear combination of the estimated coefficients of the logistic model and the new coding as follows:

SCORE $=-0.043 \times$ age $+1.049 \times$ abdominal pain $+2 \mathrm{x}$ thoracic pain $+2.109 \mathrm{x}$ skin rash $+1.747 \mathrm{x}$ positive family history

The score will be -0.043 when a patient is over 60 years of age, has a negative family history for periodic or recurrent fever episodes and presents no symptoms other than fever. It will be 22.078 when a patient is less than 10 years of age, has a positive family history for recurrent fever episodes and has all the symptoms with the highest frequency. Standardization of scores allows the probability of having a mutation to be expressed as a percentage, and thus facilitates interpretation of the results obtained. A score greater than $24 \%$ ( $>5.309$ points) identifies patients at high risk of carrying mutations 
Table II. Estimations and p values from univariate and multivariate logistic regression analyses.

\begin{tabular}{lcccc}
\hline & \multicolumn{2}{c}{ Univariate Analysis } & \multicolumn{2}{c}{ Multivariate Analysis } \\
\cline { 2 - 5 } Independent variable & OR (95\% CI) & p value & OR (95\% CI) & p value \\
\hline Abdominal pain & $2.002(1.264-3.170)$ & 0.003 & $2.853(1.057-7.701)$ & 0.038 \\
Thoracic pain & $2.012(1.301-3.112)$ & 0.002 & $7.390(2.246-24.318)$ & 0.002 \\
Skin Rash & $1.845(1.227-2.772)$ & 0.003 & $8.241(2.052-33.100)$ & 0.003 \\
Musculoskeletal involvement & $0.948(0.633-1.418)$ & 0.793 & & \\
Ocular involvement & $0.761(0.465-1.246)$ & 0.278 & & \\
Periorbital edema & $0.917(0.535-1.572)$ & 0.752 & & \\
Headache & $0.913(0.586-1.422)$ & 0.686 & & \\
Lymphadenopathy & $0.96(0.633-1.456)$ & 0.848 & & \\
Age at onset $(>16$ yrs) & $0.677(0.457-1.004)$ & 0.052 & $0.958(0.917-1.000)$ & 0.050 \\
Positive family history & $2.074(1.351-3.184)$ & 0.001 & $5.738(1.649-19.968)$ & 0.006 \\
Duration of fever $>7$ days) & $0.629(0.416-0.95)$ & 0.027 & & \\
Oral aphtosis & $1.062(0.707-1.597)$ & 0.771 & & \\
Splenomegaly & $1.00(0.63-1.587)$ & 0.999 & & \\
\hline
\end{tabular}

OR: odds ratio; CI: confidence interval

in the genes under consideration, whereas a lower percentage score (< 5.309 points) indicates a low risk. The score cut-off value was determined as the optimal level of sensitivity and specificity, and then standardized as a percentage. To obtain a standardized score it is necessary to adjust the minimum score of -0.043 to 0 and the maximum score to 22.121 and calculate the percentage by proportions.

All patients included in the training set who had a definite genetic diagnosis were studied with regression tree classification analysis in order to identify variables with predictive value for a positive genetic test. Additionally, a univariate analysis was used to evaluate which of the variables had discriminating value for differentiating between mutations in the two genes. In spite of the fact that fever attacks lasted less than 1 week in patients with mutations in either gene, the only variable with a significantly different incidence between the two genes on univariate analysis was the duration of fever attacks $(p=0.025)$.

\section{DISCUSSION}

The list of known hereditary periodic fever syndromescurrently includes two autosomal recessive conditions FMF and hyperimmunoglobulinemia D with periodic fever syndrome (HIDS) (MKD; MIM no.\#260920), and a group of autosomal dominant diseases, including TRAPS and the cryopyrin associated periodic syndromes (CAPS).

Most of these diseases typically manifest in the pediatric population, ranging from the first hours to the first decade of life, while a limited number of patients experience disease onset during adulthood. Onset of HIDS is typically in the first year of life, and genetic testing is unnecessary in patients who have their first fever attack at age $\geq 5$ years (12). 
Table III. Variables included in the final model for the calculation of the diagnostic score.

\begin{tabular}{|c|c|c|c|}
\hline Variable & Coding & Beta & Diagnostic Score \\
\hline Age at Onset & Age class* & -0.043 & $-0.043 \times$ age class $*$ \\
\hline Abdominal & Never $=0$ & 1.049 & Never $=0$ \\
\hline \multirow[t]{3}{*}{ pain } & Sometimes $=1$ & & Sometimes $=1.049$ \\
\hline & Often $=2$ & & Often $=2.098$ \\
\hline & Always $=3$ & & Always $=3.147$ \\
\hline \multirow[t]{4}{*}{ Thoracic pain } & Never $=0$ & 2.000 & Never $=0$ \\
\hline & Sometimes $=1$ & & Sometimes $=2$ \\
\hline & Often $=2$ & & Often $=4$ \\
\hline & Always $=3$ & & Always $=6$ \\
\hline \multirow[t]{4}{*}{ Skin Rash } & Never $=0$ & 2.109 & Never $=0$ \\
\hline & Sometimes $=1$ & & Sometimes $=2.109$ \\
\hline & Often $=2$ & & Often $=4.218$ \\
\hline & Always $=3$ & & Always $=6.327$ \\
\hline Positive family & Negative $=0$ & 1.747 & Negative $=0$ \\
\hline history & Positive $=1$ & & Positive $=1.747$ \\
\hline
\end{tabular}

* Age classes (pt): 7, <10 yrs; 6, 11-20 yrs; 5, 21-30 yrs; 4, 31-40 yrs; 3, 41-50 yrs;

2, 51-60 yrs; $1,>60$ yrs

CAPS are also characterized by onset in early childhood and, in addition, their chronic disease course and distinguishing clinical features often help to establish a differential diagnosis (13-14). Since our study involved mainly adult patients, only FMF and TRAPS were considered.

Late-onset FMF is fairly rare, with $14-30 \%$ of FMF patients manifesting the disease in late adulthood (6-7). Advanced age, even up to age 65, is not an exclusion criteria, as we recently reported (15). Adult-onset FMF seems to be related to lowpenetrance mutations and, although patients may experience milder disease, generally the clinical manifestations are similar to those of younger 

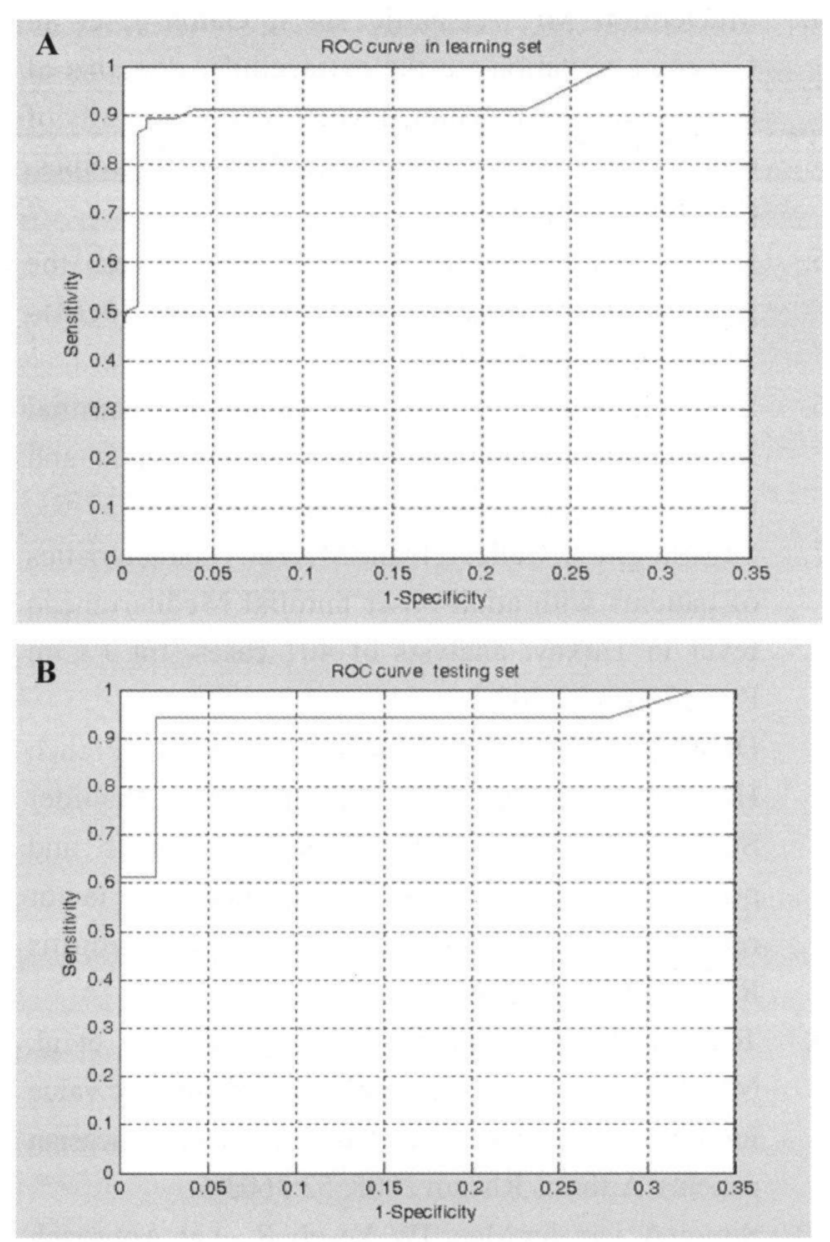

Fig. 1. A) ROC curve for the 40 patients included in the training set, indicating a sensitivity of 0.912 , a specificity of 0.960 and an accuracy of 0.955. B) ROC curve for the 70 patients included in the validation set, indicating $a$ sensitivity of 0.944 , a specificity of 0.939 and an accuracy of 0.938 .

patients, except that arthritis and erysipelas-like erythema occur at significantly lower frequencies in adults (7).

Adult onset of TRAPS, up to the age of 63, has also been described $(8,16)$. As with FMF, it is frequently related to low-penetrance mutations $(8,16-17)$. Patients with adult-onset TRAPS may be characterized by a phenotype that mimics FMF in the duration of inflammatory attacks, which may last less than 1 week (18). In addition, patients with adult-onset TRAPS may present atypical clinical manifestations, such as recurrent pericarditis as the sole clinical manifestation (8), thus mimicking autoimmune disorders $(8,18-19)$. Recurrent pericarditis is not rare, and we recently proposed clinical criteria for identifying patients who should be tested for genetic mutations of the TNFRSFIA gene (20-21). Recently, the hypothesis that recurrent pericarditis may belong to the group of autoinflammatory as opposed to autoimmune diseases has been raised (22-23), however more basic and clinical research into the etiology of this common and troublesome disease is needed.

In addition to the clinical criteria that we suggested previously (20-21), our diagnostic score may serve as an evidence-based guideline for the diagnosis of patients with recurrent fever and pericarditis, identifying the few patients among them who may prove to be carriers of mutations in "autoinflammatory genes".

Regarding the order in which genes should be examined when an autoinflammatory disease is suspected, helpful indications may come from ethnicity, family history, duration of fever attacks, and the presence of peculiar clinical manifestations. Nevertheless, perhaps more in adulthood than in childhood, the 2 disorders are largely overlapping, and it is often difficult to decide which gene should be screened first. Ethnicity may not prove useful, since patients of Mediterranean origin affected with TRAPS are commonly observed, and may be characterized by a phenotype that mimics FMF (18) in terms of fever duration, as we observed in most of the TRAPS patients enrolled in this study (Table I). This wide variability in the clinical presentation of TRAPS may lead to incorrect clinical suspicions, wrong diagnosis and improper management.

Recently, a diagnostic score for improving genetic diagnosis of recurrent periodic fever in children under the age of 10 has been proposed that increases the probability of obtaining positive results in genetic testing and reduces the cost of molecular analysis (24). However, this pediatric diagnostic score could not distinguish our genetically-positive adult patients from genetically-negative patients: most of the subjects who carried mutations in the TNFRSF $1 A$ gene were identified as low-risk patients. This was probably related to the fact that most of the patients carrying mutations in the TNFRSFIA gene were characterized by incomplete disease.

Federici et al. also performed a study on a large 
group of adult patients, and suggested that meeting FMF criteria (25) and being of Mediterranean origin should be considered clear indications for recommending molecular analysis of the $M E F V$ gene. The authors also underlined that in the absence of these features or in the presence of a negative genetic test for mutations in the $M E V F$ gene, the next gene to be screened should be chosen on the basis of expert advice (25). Of note, we could not apply these recommendations to our cohort since all of our patients were of Italian origin, thus multivariate analysis could not evaluate the ethnic origin of patients among the possible discriminating variables. Nevertheless, ethnic origin should obviously be taken into consideration when patients from populations with a high prevalence of mutations of a certain gene are examined (25). The same is true in the case of patients who meet diagnostic criteria for FMF (26).

In conclusion, we propose a diagnostic score for evaluating adults with recurrent fever attacks that may be useful for identifying those at high risk for carrying mutations in the $M E F V$ and TNFRSF1A genes. In particular, we have identified abdominal and thoracic pain, skin manifestations, young age at onset and positive family history of periodic fever syndromes as being variables that appear to correlate strongly with the probability of detecting gene mutations in $M E F V$ or TNFRSFIA in adults, thus improving the evaluation of patients with suspected autoinflammatory disorders. Nevertheless, further evaluation including more patients, different populations and using longitudinal studies are needed to verify the sensitivity and specificity of the proposed score.

\section{REFERENCES}

1. Masters SL, Lobito AA, Chae JJ, Kastner DL. Recent advances in the molecular pathogenesis of hereditary recurrent fevers. Curr Opin Allergy Clin Immunol 2006; 6:428-33.

2. The French FMF Consortium. A candidate gene for familial Mediterranean fever. Nat Genet 1997; 17: 25-31.

3. The International FMF Consortium. Ancient missense mutations in a new member of the RoRet gene family are likely to cause familial Mediterranean fever. Cell 1997; 90:797-807.
4. McDermott MF, Aksentijevich I, Galon J, et al. Germline mutations in the extracellular domains of the $55 \mathrm{kDa}$ TNF receptor, TNFR 1 , define a family of dominantly inherited autoinflammatory syndromes. Cell 1999; 97:133-44.

5. Kastner DL. Familial Mediterranean fever: the genetics of inflammation. Hosp Pract 1998; 33:13134, 9-40, 43-46 passim.

6. Sohar E, Gafni J, Pras M, Heller H. Familial Mediterranean fever. A survey of 470 cases and review of the literature. Am J Med 1967; 43:227-53.

7. Sayarlioglu M, Cefle A, Inanc M, et al. Characteristics of patients with adult-onset familial Mediterranean fever in Turkey: analysis of 401 cases. Int J Clin Pract 2005; 59:202-5.

8. Dodé $\mathrm{C}$, André $\mathrm{M}$, Bienvenu $\mathrm{T}$, et al. French Hereditary Recurrent Inflammatory Disorder Study Group. The enlarging clinical, genetic, and population spectrum of tumor necrosis factor receptor-associated periodic syndrome. Arthritis Rheum 2002; 46:2181-8.

9. Tchernitchko D, Moutereau S, Legendre $M$, et al. MEFV analysis is of particularly weak diagnostic value for recurrent fevers in western European Caucasian patients. Arthritis Rheum 2005; 52:3603-5.

10. Simon A, van der Meer JW, Vesely R, et al. Approach to genetic analysis in the diagnosis of hereditary autoinflammatory syndromes. Rheumatology (Oxford) 2006; 45:269-73.

11. Touitou I, Lesage S, McDermott M, et al. Infevers: An evolving mutation database for auto-inflammatory syndromes. Human Mutation 2004; 24:194-8.

12. Steichen $O$, van der Hilst J, Simon A, Cuisset L, Grateau G. A clinical criterion to exclude the hyperimmunoglobulin D syndrome (mild mevalonate kinase deficiency) in patients with recurrent fever. $J$ Rheumatol 2009; 36:1677-81.

13. Neven B, Callebaut I, Prieur AM, et al. Molecular basis of the spectral expression of CIAS1 mutations associated with phagocytic cell-mediated autoinflammatory disorders CINCA/NOMID, MWS, and FCU. Blood 2004; 103:2809-15.

14. Caroli F, Pontillo A, D'Osualdo A, et al. Clinical and genetic characterization of Italian patients affected by CINCA syndrome. Rheumatology (Oxford) 2007; 46:473-8. 
15. Cantarini L, Capecchi PL, Lucherini OM, Laghi Pasini F, Galeazzi M. Familial Mediterranean fever diagnosed in an elderly patient. Clin Exp Rheumatol 2010; $28(\mathrm{~S}) 591$.

16. Hull KM, Drewe E, Aksentijevich I, et al. The TNF receptor-associated periodic syndrome (TRAPS): emerging concepts of an autoinflammatory disorder. Medicine (Baltimore) 2002; 81:349-68.

17. Ravet $\mathrm{N}$, Rouaghe $\mathrm{S}$, Dodé $\mathrm{C}$, et al. Clinical significance of $\mathrm{P} 46 \mathrm{~L}$ and $\mathrm{R} 92 \mathrm{Q}$ substitutions in the tumour necrosis factor superfamily $1 \mathrm{~A}$ gene. Ann Rheum Dis 2006; 65:1158-62.

18. Dodé C, Papo T, Fieschi C, et al. A novel missense mutation $(\mathrm{C} 30 \mathrm{~S})$ in the gene encoding tumor necrosis factor receptor 1 linked to autosomal-dominant recurrent fever with localized myositis in a French family. Arthritis Rheum 2000; 43:1535-42.

19. Kiss MH, Magalhães CS. Autoinflammatory Diseases: Mimics of Autoimmunity or Part of its Spectrum? Case Presentation. J Clin Immunol 2008; 28(S):84-9.

20. Cantarini L, Lucherini OM, Cimaz R, et al. Idiopathic recurrent pericarditis refractory to colchicine treatment can reveal tumor-necrosis factor associated periodic syndrome. Int J Immunopathol Pharmacol 2009; 22:1051-8.
21. Cantarini L, Lucherini OM, Baldari CT, Laghi Pasini F, Galeazzi M. Familial clustering of recurrent pericarditis may disclose tumor necrosis factor receptor-associated periodic syndrome. Clin Exp Rheumatol 2010; 28:405-7.

22. Cantarini L, Imazio $\mathrm{M}$, Brucato A, Lucherini $\mathrm{OM}$, Galeazzi M. Innate versus acquired immune response in the pathogenesis of recurrent idiopathic pericarditis. Autoimmun Rev 2010; 9:436-40.

23. Picco P, Brisca G, Traverso F, Loy A, Gattorno M, Martini A. Successful treatment of idiopathic recurrent pericarditis in children with interleukin1 beta receptor antagonist (anakinra): an unrecognized autoinflammatory disease? Arthritis Rheum 2009; 60:264-8.

24. Gattorno M, Sormani MP, D'Osualdo A, et al. A diagnostic score for molecular analysis of hereditary autoinflammatory syndromes with periodic fever in children. Arthritis Rheum 2008; 58:1823-32.

25. Federici L, Rittore-Domingo C, Kone-Paut I, et al. A decision tree for genetic diagnosis of hereditary periodic fever in unselected patients. Ann Rheum Dis 2006; 65:1427-32.

26. Livneh A, Langevitz P, Zemer D, et al. Criteria for the diagnosis of familial Mediterranean fever. Arthritis Rheum 1997; 40:1879-85. 